\title{
Editorials
}

\section{Post-traumatic stress disorder:}

\author{
what does NICE guidance mean for primary care?
}

\section{INTRODUCTION}

Post-traumatic stress disorder (PTSD) may develop following exposure to an extremely threatening or horrific event or series of events.' Traumatic events which may lead to PTSD include: serious accidents; physical or sexual assault; abuse; workrelated exposure to trauma; serious health problems or childbirth experiences; war and conflict; or torture. PTSD can affect people of any age. The estimated population prevalence of PTSD in adults in the UK is around one in 20 (4.4\%), with similar rates observed between men and women. ${ }^{2}$ The symptoms of PTSD can cause considerable distress and may substantially impede social, educational, and occupational functioning. ${ }^{2}$ People with PTSD may also develop coexisting conditions including substance misuse and depression. ${ }^{3-5}$ PTSD is a treatable disorder, even for people who present many years after trauma. However, effective treatment can only take place if the disorder is recognised. GPs are frequently the first healthcare professional that a patient consults. A new National Institute for Health and Care Excellence (NICE) guideline on the assessment and management of people with PTSD 6 has important, practical messages which should support GPs in the recognition of PTSD and the facilitation of timely access to effective PTSD treatments. In this editorial we outline the current challenges around the recognition of PTSD in primary care and the onward referral for treatment, and highlight the key NICE guideline recommendations relevant to GPs and what can be done to promote access to treatment for people with PTSD.

\section{IDENTIFICATION AND DIAGNOSIS}

PTSD can present with a range of symptoms. In adults, most common PTSD symptoms include: re-experiencing the traumatic event(s) in the form of distressing memories, flashbacks, or nightmares; avoidance of trauma-related reminders; and hypervigilance. Children, particularly when younger, may re-enact the traumatic experience through repetitive play or have frightening dreams with no recognisable content. The NICE guideline recommends that GPs should take responsibility for assessment and the initial coordination of care for people with clinically important symptoms of PTSD presenting in primary

\section{GPS should consider the diagnosis of PTSD proactively, and ask about experience of trauma}

care. However, national survey statistics suggest there are significant barriers to people with PTSD receiving a diagnosis, for instance, only one in eight of those who screened positive for PTSD had been diagnosed by a health professional. ${ }^{2}$ Potential reasons for this low recognition rate include patient factors, time constraints, and comorbidity with other psychological or physical conditions that complicate differential diagnosis. ${ }^{7}$ A potentially relevant patient factor is that people with PTSD may avoid discussing their problems, for instance, $40 \%$ of those who screened positive for PTSD had not spoken with a GP about a mental or emotional problem in the last year. ${ }^{2}$ This may not be surprising given that one of the core symptoms of PTSD is avoidance.

The NICE guideline clearly outlines the signs and symptoms that should prompt assessment for PTSD. Importantly, the guideline highlights that GPs should consider the diagnosis of PTSD proactively, and ask about experience of trauma. For example, patients with PTSD may seek care for the physical health consequences of trauma or may present with a range of medically unexplained physical symptoms such as gastrointestinal difficulties, pain syndromes, or headaches. PTSD may also be at the root of more general functional impairment, relationship difficulties, or problem drinking. Therefore, it is worth enquiring whether patients presenting with such problems have ever experienced a traumatic event, which may have been some time ago, after which their difficulties started.

The NICE guideline emphasises that identifying PTSD in children presents particular difficulties, partly because children may not complain directly of PTSD symptoms, such as re-experiencing or avoidance. However, difficulties can be ameliorated by asking children directly about the presence of PTSD symptoms rather than relying solely on the parent or carer for information.

GPs should be aware that many people do not develop PTSD after a traumatic event, even without intervention. Early distress symptoms, often seen within the first days or weeks of trauma, include poor sleep, rumination, and anxiety. Where these are transient and do not substantially impair day-to-day function they should be considered as a normal reaction to an abnormal event'. However, persistent distress symptoms associated with functional impairment, if still present 1 month after an incident, should raise the possibility of a PTSD diagnosis. The NICE guideline recommends active monitoring for people with subthreshold symptoms of PTSD within 1 month of a traumatic event, to judge whether further intervention is required. It is also important for GPs to provide information about common reactions to traumatic events and the symptoms of PTSD in order to increase awareness. People with PTSD should ideally not be exposed to further trauma prior to treatment and this may be communicated to employers in a fit note.

\section{TREATMENT}

There are effective treatments for PTSD and the NICE guideline recommends that psychological treatment should be offered, namely, trauma-focused cognitive behavioural therapy (CBT), eye movement desensitisation and reprocessing (EMDR), or supported trauma-focused computerised CBT la facilitated selfhelp intervention). The NICE guideline also recommends that trauma-focused CBT should be available from as early as within the first month of trauma. Implementing the recommendations from the NICE guideline, in particular the 1 month target to psychological treatment for people presenting in the immediate aftermath of trauma, is not going to be without challenges; a national survey found that only $24 \%$ of people screening positive for PTSD were receiving psychological treatment, and people with PTSD were far more likely than the rest of the population to have requested, but not been given, 
particular treatment $16 \%$ compared with $1 \%)^{2}$ The GP can be particularly helpful in addressing some of the barriers to accessing treatment by giving some key messages, including reassuring those with probable PTSD that the condition is treatable, providing information about treatment options, and making people aware that they can self-refer through Improving Access to Psychological Therapies (IAPT) pathway. The latter may also be important in terms of addressing some of the issues with reluctance to disclose, as people may want to avoid telling other individuals their story, and may prefer restricting disclosure only to a health professional who can offer them a course of treatment. ${ }^{7}$ The NICE guideline recognises and recommends that further research is needed on stepped care approaches to investigate whether less intensive and more accessible forms of treatment can deliver equivalent therapeutic outcomes.

Research suggests that medications, especially selective serotonin reuptake inhibitors (SSRIs), appear to be more commonly prescribed than recommended

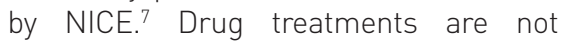
recommended as first-line treatment for PTSD. Importantly, benzodiazepines are contraindicated in PTSD and should not be used for prevention or treatment. An SSRI or venlafaxine should only be considered where an adult with a diagnosis of PTSD has a preference for drug treatment. Antipsychotic treatment should also only be considered as a last resort in the context of disabling symptoms and behaviours, and should only be prescribed in a specialist setting or after consultation with a specialist. The issue of drug treatment is confounded by comorbidity with depression and problems with differential diagnosis. However, it is important to note that the NICE guideline recommends that for people presenting with PTSD and depression, the PTSD should usually be treated first because the depression will often improve with successful PTSD treatment. Depression should only be treated first if it is severe enough to make psychological treatment of the PTSD difficult, or there is a risk of harm to self or others. The NICE guideline also emphasises that people should not be excluded from treatment based solely on comorbid alcohol or substance misuse problems and should be referred for specialist assessment and PTSD treatment. Referral for treatment should also not be delayed or withheld due to legal proceedings.

\section{CONCLUSION}

People with PTSD are often apprehensive about making contact with healthcare services; are often anxious or feel ashamed; believe that PTSD is untreatable; and may not know what interventions and help are available. However, people with PTSD do come into contact with healthcare services, for instance, $61 \%$ of those screening positive for PTSD had contacted some form of healthcare service during the last year with the vast majority speaking to their GP. ${ }^{2}$ A key way in which the GP can help people with PTSD to recover is to have the diagnosis in mind and identify those whose distress symptoms are persistent (more than a month after a traumatic event) and associated with functional impairment. Such individuals should be referred on for specialist care which is likely to include trauma-focused CBT or EMDR.

\section{Odette Megnin-Viggars,}

Senior Systematic Reviewer, Centre for Outcomes Research and Effectiveness, Research Department of Clinical, Educational, \& Health Psychology, University College London; National Guideline Alliance, Royal College of Obstetricians and Gynaecologists, London.

\section{Ifigeneia Mavranezouli,}

Senior Health Economist, Centre for Outcomes

Research and Effectiveness, Research Department of Clinical, Educational, \& Health Psychology, University College London; National Guideline Alliance, Royal College of Obstetricians and Gynaecologists, London.

\section{Neil Greenberg,}

Professor of Defence Mental Health, King's College London, London.

\section{Steve Hajioff}

Director of Public Health, London Borough of Hillingdon; Honorary Senior Lecturer, Department of Public Health \& Primary Care, Imperial College London, London.

\section{Jonathan Leach,}

GP, Davenal House Surgery, Bromsgrove; NHS England Medical Director for Armed Forces and Veterans Health; Joint Honorary Secretary, RCGP, London.

\section{Provenance}

Freely submitted; externally peer reviewed.

\section{Competing interests}

The views expressed in this editorial are those of the authors and not necessarily those of NICE.

\section{Acknowledgements}

The guideline referred to in this article was produced by the National Guideline Alliance (NGA) for the National Institute for Health and Care Excellence (NICE).

DOI: https://doi.org/10.3399/bjgp19X704189

\section{ADDRESS FOR CORRESPONDENCE}

\section{Odette Megnin-Viggars}

Centre for Outcomes Research and Effectiveness, University College London, 1-19 Torrington Place, London WC1E 7HB, UK.

Email: o.megninAucl.ac.uk

\section{REFERENCES}

1. World Health Organization. International statistical classification of diseases and related health problems. 2018. https://icd. who.int/browse11/l-m/en laccessed 7 Jun 2019)

2. Fear NT, Bridges S, Hatch S, et al. Posttraumatic stress disorder. In: McManus S, Bebbington P, Jenkins S, Brugha T, eds. Mental health and wellbeing in England: adult psychiatric morbidity survey 2014. Leeds: NHS Digital, 2016: 106-130.

3. Brady KT, Killeen TK, Brewerton T, Lucerin S. Comorbidity of psychiatric disorders and posttraumatic stress disorder. J Clin Psychiatry 2000; 61(Suppl 7): 22-32.

4. Debell F, Fear NT, Head M, et al. A systematic review of the comorbidity between PTSD and alcohol misuse. Soc Psychiatry Psychiatr Epidemiol 2014; 49(9): 1401-1425.

5. Rytwinski NK, Scur MD, Feeny NC Youngstrom EA. The co-occurrence of major depressive disorder among individuals with posttraumatic stress disorder: a metaanalysis. J Trauma Stress 2013; 26(3): 299-309.

6. National Institute for Health and Care Excellence. Post-traumatic stress disorder. NG116. 2018. https://www.nice.org.uk/ guidance/ng116 laccessed 7 Jun 2019).

7. Ehlers A, Gene-Cos N, Perrin S. Low recognition of post-traumatic stress disorder in primary care. London J Prim Care 2009; 2(1): 36-42 